\title{
NUCLEAR ACTIVITY IN THE HICKSON COMPACT GROUPS OF GALAXIES
}

\author{
M. SHIMADA, S. NISHIURA, Y. OHYAMA, T. MURAYAMA AND \\ Y. TANIGUCHI \\ Astronomical Institute, Tohoku University \\ Aramaki, Aoba, Sendai 980-77, JAPAN
}

In order to study environmental effects on the nuclear activity in galaxies, we have been conducting a spectroscopic study of Hickson Compact Groups of galaxies (HCGs, Hickson 1982) which are the densest agglomeration of galaxies. We obtained nuclear spectra of 62 galaxies in 29 HCGs in the spectral range $6200-7000 \AA$ with the $188 \mathrm{~cm}$ telescope at Okayama Astrophysical Observatory. These spectra were classified into the three types by using the emission line ratio [NII] $\lambda 6583 / \mathrm{H} \alpha$; (1) AGN: [NII] $\lambda 6583 / \mathrm{H} \alpha>0.6,(2) \mathrm{HII}$ nuclei: $[\mathrm{NII}] \lambda 6583 / \mathrm{H} \alpha<0.6$, and (3) Absorption: no emission line. We compared the nuclear activity of galaxies in HCGs with that of nearby galaxies (Ho 1996; Ho, Filippenko \& Sargent 1997) which provides a representative sample of field galaxies. In early-type spirals (Sa-Sbc), the fraction of HII nuclei in HCGs is smaller than that in the field galaxies, while the fraction of absorption in HCGs is larger than that in field galaxies. On the other hand, in early-type galaxies (E-S0a) and late-type spirals (Sc-P), we found little difference in the nuclear activity between HCGs and field galaxies.

\section{References}

Hickson, P. 1982, ApJ, 255, 382

Ho, L.C. 1996, in The Physics of LINERS in View of Recent Observations, ASP Conf. Ser., 103,103

Ho, L.C., Filippenko, A.V., \& Sargent, W.L.W. 1997, ApJ, 487, 568 